\title{
Interpersonal Touch Buffers Pain Sensitivity in Romantic Relationships but Heightens Sensitivity between Strangers and Friends
}

\author{
Kory Floyd ${ }^{1,{ }^{*}}$, Colter D. Ray ${ }^{2}$, Lisa J. van Raalte ${ }^{3}$, James B. Stein ${ }^{4}$, Mark Alan Generous ${ }^{5}$ \\ ${ }^{1}$ Department of Communication, University of Arizona, Tucson, USA \\ ${ }^{2}$ School of Communication, San Diego State University, San Diego, USA \\ ${ }^{3}$ Department of Communication Studies, Sam Houston State University, Huntsville, USA \\ ${ }^{4}$ Hugh Downs School of Human Communication, Arizona State University, Tempe, USA \\ ${ }^{5}$ Department of Communication Studies, California State Polytechnic University, Pomona, USA \\ *Corresponding author: koryfloyd@email.arizona.edu
}

\begin{abstract}
A variety of evidence suggests that interpersonal behaviors such as touch can have pain-alleviating effects on recipients. Less well understood is how touch affects sensitivity to pain in the first place, and whether its effects depend on the nature of the relationship in which it occurs. In the present experiment, 55 adults who had not been diagnosed with a pain disorder were exposed to a cold pressor pain induction with an opposite-sex stranger, platonic friend, or romantic partner who either touched their shoulders, was present in the room without touching them, or was absent during the cold pressor. The outcome variable was the self-reported level of pain induced by the stimulus at the moment when the stimulus became painful (i.e., pain sensitivity). Compared to presence or absence, touch dampened pain sensitivity for romantic partners, allowing them to tolerate more intensity before the stimulus became painful. Touch had the opposite effect for friends and strangers, heightening their sensitivity to pain.
\end{abstract}

Keywords: touch, pain, cold pressor, sensitivity

Cite This Article: Kory Floyd, Colter D. Ray, Lisa J. van Raalte, James B. Stein, and Mark Alan Generous, "Interpersonal Touch Buffers Pain Sensitivity in Romantic Relationships but Heightens Sensitivity between Strangers and Friends.” Research in Psychology and Behavioral Sciences, vol. 6, no. 1 (2018): 27-34. doi: 10.12691/rpbs-6-1-4.

\section{Introduction}

Pain is a significant and persistent health problem for millions. In the United States alone, an estimated 126 million adults experience recurring pain, and 23.4 million experience significant pain, according to research from the U.S. National Institutes of Health [1]. That pain translates into severe economic losses, with workers losing an average of 4.6 hours of productivity per week due to pain, a loss equivalent to an estimated $\$ 61.2$ billion annually [2]. Pain not only is discomforting; it can also severely impede quality of life. The Voices of Chronic Pain Survey conducted by the American Pain Foundation reported that chronic pain is frequently comorbid with depression (77\%), difficulty concentrating (70\%), reduced energy (74\%), and impaired sleep (86\%) [3].

Pain can often be effectively managed with pharmaceutical therapies and with behaviors such as exercise [4]. In addition, however, a robust empirical literature attests to the health-promotive and pain-alleviating effects of personal relationships and relational interaction. In terms of health benefits, for instance, research shows that relationship quality and emotional support protect against upper respiratory illness and infection [5]; reduce cortisol and increase oxytocin reactivity to stressors [6,7]; modulate 24-hour adrenocortical activity [8]; are associated with lower resting blood pressure [9]; and even reduce susceptibility to the common cold [10]. The present study examines the possibility that interpersonal touch in the context of such relationships between romantic partners, platonic friends, or even strangers also contributes to wellness by buffering the pain response.

This review begins with a brief definition of pain, followed by a description of research linking touch to health- and pain-related outcomes. We then identify pain sensitivity as the principal focus of the present study and offer specific hypotheses and a research question.

\section{The Experience of Pain}

The International Association for the Study of Pain defines pain as "an unpleasant sensory and emotional experience associated with actual or potential tissue damage, or described in terms of such damage" [11]. According to this definition, the potential for tissue damage-such as might be caused by injury, extreme pressure, electric shock, or exposure to extreme temperatures-underlies the experience of pain. Importantly, however, the same definition clarifies that 
"pain is always subjective," suggesting that there is no more objective way to assess pain than via self-report. Pain levels cannot be established through mere exposure to a tissue-damaging stimulus, in other words, but are based on an individual's subjective assessment of the magnitude of pain that he or she is experiencing from such a stimulus.

In clinical practice, pain levels are assessed in various ways. Some clinicians use a visual analogue scale, such as one asking patients to locate their pain level on a continuum from "no pain" to "worst possible pain” [12]. Another commonly used measure is the Wong-Baker FACES scale, which presents six faces ranging from a smiling face to a crying face and asks patients to indicate which face best represents their pain level [13]. Finally, some clinicians use a simple numerical rating scale that asks patients to identify their pain level on a scale from $1-10$ or $1-100$ [14].

A variety of methods exists for inducing pain in laboratory settings [15]. Some studies expose the skin to radiant heat [16], heated water [17], or extreme cold (e.g., the cold pressor test) [18]. Other studies generate ischemic pain by obstructing blood flow in an extremity, as occurs when a blood pressure cuff is inflated [19]. Electrical stimulation [20], application of pressure [21], and chemical exposure [22] are also used to induce pain in laboratory contexts.

Because pain is inherently subjective, two individuals may experience the same stimulus but may report that it causes different levels of pain. As we detail below, the focus of the present investigation is on the level of pain perceived at the point at which a stimulus becomes painful (which is one way of measuring an individual's pain sensitivity), and the extent to which that pain level is affected by interpersonal touch.

\subsection{Touch and Pain}

One interpersonal activity that has shown health-promotive effects is touch. Compelling evidence of the health benefits of touch comes from the extensive empirical literature on massage therapy. Tactile contact in the form of massage improves a broad range of physical conditions, including anorexia [23], asthma [24], dermatitis [25], diabetes [26], HIV [27], hypertension [28], leukemia [29], nicotine addiction [30], Parkinson's disease [31], sleep disorders [32], and stress [33].

\subsubsection{Massage Therapy}

Moreover, multiple experiments find that massage therapy reduces pain. For instance, Frey Law et al. assigned laboratory participants to receive either a deep-tissue massage, superficial touch, or no treatment [34]. During subsequent wrist extension exercises, participants in the massage group and in the touch group both experienced decreased delayed-onset muscle soreness relative to control participants. Massage therapy has also proven effective at reducing pain for postoperative patients [35], advanced cancer patients with moderate-to-severe pain [36], cardiac surgery patients [37], and for children and adolescents with chronic pain [38].

\subsubsection{Non-Massage Touch}

Touch outside of the massage therapy context has also been shown to be effective for pain reduction. For example, hand holding received by pediatric oncology patients [39] and adolescents with renal disease [40] was associated with reduced pain during medical procedures. Similarly, informal back rubs reduced pain for elderly stroke patients [41]. Multiple studies have also shown that gentle touch and skin-to-skin contact reduce the magnitude of pain responses in infants $[42,43,44]$. Hogendoorn, Kammers, Haggard, and Verstraten even found that self-touch, in the form of lightly pressing one's own hands together, reduces the neurological pain response [45]. Conversely, adults who report being deprived of interpersonal affection-including touch-also report higher levels of chronic pain [46].

\subsubsection{Potential Mechanisms Linking Pain and Touch}

Multiple potential mechanisms link touch to pain perception. Melzack and Wall's gate control theory of pain postulates that pain stimulates nerve fibers that are shorter and less myelinated (i.e., less insulated) than does touch, causing pain signals to take longer to reach the central nervous system than tactile signals - thus, touch effectively "blocks the gate" for pain signals to be processed [47].

Neurophysiological research with animals has identified a class of wide dynamic range (WDR) neurons located in the dorsal horn, which respond to both tactile and nociceptive (i.e., related to pain arising from nerve cell stimulation) inputs [48]. These WDR neurons may also play a role in generating analgesic effects from touch. Based on this observation, Mancini, Nash, Iannetti, and Haggard found that sensitivity to radiant heat was reduced by tactile stimulation [49].

Two potential biochemical mechanisms linking touch to the experience of pain are an increase in the pituitary hormone oxytocin and the release of endogenous opioids known as endorphins [50]. Oxytocin is a peptide produced by the hypothalamus and released primarily by the posterior pituitary gland [51]. When elevated in the bloodstream, it produces sensations of calm, pain relief, warmth, and suppressed stress reactivity in the hypothalamic-pituitary-adrenal axis [52,53]. In expectant mothers, oxytocin initiates uterine contractions and stimulates the milk let-down reflex [54]. Beyond its role in the childbirth process, however, oxytocin is elevated at sexual orgasm in both women and men [55,56], and is also elevated in both sexes in response to affectionate but nonsexual touch $[57,58,59,60]$. Light et al. also reported that baseline oxytocin levels in women are directly associated with the reported number of hugs received from their husbands or male partners [9], and Floyd et al. demonstrated that affectionate behavior is associated with oxytocin increases in response to stressors, which may partially account for the stress-buffering effect of affectionate behavior [7]. Ågren, Lundeberg, UvnäsMoberg, and Sato similarly reported that massage-like abdominal stroking elevated plasma oxytocin levels in rats and simultaneously increased pain tolerance [61].

A second potential biochemical mechanism implicates the activity of endogenous opioids called endorphins. Endorphins have long been recognized for their role in pain control [62]. When elevated in the bloodstream, endorphins produce an opiate-like state that has mild analgesic effects [63]. Research indicates that endorphins are particularly responsive to low-level muscular pain [64] and psychological or emotional distress [65]. As Dunbar 
suggested, endorphins project to areas of the brain well positioned to receive input from physical touch, yet direct evidence linking endorphin levels to touch in humans is lacking because endorphins can be sampled only in cerebrospinal fluid, making them difficult to assay [50].

In sum, multiple potential mechanisms-alone or in combination-may connect the interpersonal experience of touch to pain and pain sensitivity, explicated next.

\subsection{Pain Sensitivity}

One way to appreciate the potential effects of touch on pain perception is to examine its association with sensitivity to pain. Pain sensitivity is often measured as a duration threshold, which represents the amount of time that elapses from the initiation of a stimulus (such as a pain induction) to the point at which the stimulus is perceived as painful [66]. However, sensitivity can also be operationalized as an intensity threshold, which represents the intensity of pain being experienced at the moment when the stimulus is perceived as painful [49]. Instead of asking "at what point in time does the stimulus begin to hurt?”, conceiving of pain sensitivity as an intensity threshold asks "how much pain is present when the stimulus becomes painful?" According to this conceptualization, higher sensitivity is indexed by lower pain scores because a lower intensity of pain is experienced as painful. In contrast, when an individual can tolerate higher pain intensity before experiencing a stimulus as painful, this corresponds to lower sensitivity.

\subsubsection{Hypotheses and Research Question}

Extant evidence supports the possibility that touch has a protective effect on pain sensitivity, meaning that receiving touch allows an individual to tolerate higher pain intensity before experiencing a stimulus as painful, relative to receiving no touch (H1). Importantly, however, Coan, Schaefer, and Davidson found that the beneficial effects of touch were moderated by the nature of the relationship in which touch occurred [67]. Specifically, Coan et al.’s participants-all married women-benefited (in terms of attenuated responses to threat) more when holding someone's hand than when not holding someone's hand (consistent with the present study's H1), but they benefited more when holding the hand of their husband than the hand of a stranger. On this basis, we further predict that the protective effect of touch on pain sensitivity is greater for romantic partners than for other relationships (H2).

In addition, several studies have demonstrated differences between women and men in pain sensitivity $[68,69]$. These findings raise the possibility that the protective effect of touch varies by the biological sex of the participant, an issue we address in the form of a research question (RQ1).

\section{Method}

\subsection{Participants}

Participants $(N=55)$ were 28 women and 27 men ranging in age from 18 to 49 years $(M=23.25, S D=6.69)$. The majority of the participants identified as White/Caucasian (70.9\%), whereas 14.5\% identified as Hispanic/Latino(a),
12.7\% as Black/African American, 9.1\% as Asian/Pacific Islander, and $1.8 \%$ as Native American (5.5\% reported having other racial or ethnic backgrounds). (Percentages sum to $>100$ because some participants reported more than one racial or ethnic background.)

\subsection{Procedure}

\subsubsection{Recruitment and Prescreening Procedure}

Participants were recruited primarily from undergraduate communication courses at a large university in the southwest region of the United States. A smaller number of participants was recruited through the authors' personal networks. Students were offered extra credit in exchange for their participation in what we, following DeWall and Baumeister, described to them as a study of personality and physical sensitivity [70]. In support of the cover story, prospective participants were asked to complete and submit an online questionnaire that, in addition to the prescreening questions, included a standard assessment of the Big 5 personality traits (detailed below).

To qualify for participation in the study, prospective participants had to: (a) be 18 years of age or older, (b) speak and read English fluently, and (c) have not been diagnosed with or treated for fibromyalgia or a chronic pain disorder. A total of 162 prospective participants completed and submitted a prescreening questionnaire. Of these, $98.1 \%$ met all the inclusion criteria, with the remaining prospective participants being disqualified because of diagnosis or treatment for fibromyalgia. Women and men were equally likely to be qualified for the study.

\subsubsection{Laboratory Procedure}

All laboratory sessions were conducted in the Health Communication Laboratory of the university's school of communication. The university's bioscience institutional review board approved all lab procedures. Participants who met eligibility criteria were contacted by researchers to schedule an appointment with the Health Communication Laboratory. Some participants $(n=37)$ were also instructed to bring either an opposite-sex friend or an opposite-sex romantic partner. Conversely, other participants $(n=18)$ were informed they would be paired with an opposite-sex stranger during their laboratory session.

Upon arrival, both the participant and companion were given informed consent forms to read, sign, and date. After consent was obtained from both the participant and companion, the companion was led to a separate room. At this time, both the participant and companion independently read the experimental instructions and completed a short survey that included a manipulation check. While the companion waited in the next room, the experimenter measured the participant's height, weight, heart rate, and blood pressure. The experimenter then retrieved the companion from the waiting room and orally reviewed each person's instructions for the impending cold pressor test, detailed next.

\subsubsection{Pain Induction}

A cold pressor test was used to induce pain [7,71]. Participants were instructed to submerge their left forearm into a 3-gal galvanized bucket filled with ice water and 
multiple gel refrigerant packs (water temperature at session onset $M=38.43^{\circ} \mathrm{F}, S D=3.25$ ) and place the palm of their hand flat against the bottom of the bucket.

\subsubsection{Partner Condition}

Each participant was paired with either a romantic partner, a platonic friend, or a stranger of the opposite sex for the laboratory session.

\subsubsection{Touch Manipulation}

During the cold pressor procedure, the companion was randomly assigned to stand behind the participant and rest his or her hands on the participant's shoulders, to be present in the room during the cold pressor test by standing behind the participant but not touching him or her, or to be absent. If the companion was present or touching the participant during the cold pressor test, he or she was instructed not to converse with the participant or communicate nonverbally by rubbing or massaging the participant's shoulders.

\subsection{Measures}

\subsubsection{Prescreening Measures}

The screening survey included a series of self-report measures related to personality, affection, and loneliness (investigated as potential control variables) as well as the study criteria questions. Unless otherwise noted, the scales described herein were measured using 7-point Likert items (1 = strongly disagree and 7 = strongly agree $)$.

Trait affectionate communication was measured using the Trait Affection Scale-Given (TAS-G) and the Trait Affection Scale-Received (TAS-R) [72]. Both scales have been extensively validated [73]. For the present study, Cronbach's alpha coefficients for both scales were adequate (TAS-G $\alpha=.86$; TAS-R $\alpha=.87$ ).

Loneliness was measured using the UCLA Loneliness Scale [74,75]. The scale has been extensively validated and typically yields a coefficient $\alpha$ between .84 and .94 . The internal reliability in the present study was also within this range $(\alpha=.94)$.

Personality traits were measured using the Big Five Inventory [76,77]. The BFI consists of 44 items measuring extraversion, agreeableness, conscientiousness, neuroticism, and openness to experiences. The BFI has been validated extensively [77]. In the present study, all five personality trait measures had acceptable reliabilities (extraversion $\alpha=$.84; agreeableness $\alpha=$.78; conscientiousness $\alpha=.75$; neuroticism $\alpha=.79$; openness $\alpha=.84$ ).

Opposite-sex touch avoidance was measured using Andersen and Leibowitz's touch avoidance measure, version two (TAM2) scale [78]. Items measure the participant's affective perceptions of both giving and receiving touch from people of the opposite sex on a scale from 1 (strongly disagree) to 9 (strongly disagree). Multiple studies have confirmed the TAM2 as a valid and reliable measure of opposite-sex touch avoidance [79]. The reliability coefficient was adequate $(\alpha=.89)$.

\subsubsection{Laboratory Measures}

Upon arrival in the laboratory, participants had their height and weight recorded by a research assistant to calculate body mass index for use as a potential control variable. After a period of acclimation, the research assistant also took manual readings of participants' pulse rate $(M=73.33$ beats per minute, $S D=11.07)$, systolic blood pressure $(M=124.30 \mathrm{mmHg}, S D=9.15)$, and diastolic blood pressure $(M=81.53 \mathrm{mmHg}, S D=8.31)$ to confirm that all were within normal limits before beginning the experimental procedure.

Immediately upon the participants immersing their arm in the ice water, the experimenter activated a digital stopwatch. Participants were instructed to say "now" when pain was initially felt, and to rate the severity of the pain at that moment. This rating was verbalized by the participant as a number, ranging from 1 (not painful at all) to 9 (extremely painful) and recorded by the experimenter.

\subsection{Manipulation Check}

A manipulation check was included to determine if the participant and partner were indeed strangers, oppositesex friends, or opposite-sex romantic partners, depending on the experimental condition. This was accomplished by asking each participant to circle on a paper survey whether the other person was a romantic partner, friend, or stranger. Moreover, each participant and companion was also asked rate on a 1 to 9 scale how well they knew the other person, with higher numbers indicating greater familiarity.

\section{Results}

\subsection{Manipulation Check}

In all but one case, both partners in the dyad identified their relationship in the same way (whether strangers, friends, or romantic partners) and in a manner consistent with the experimental relationship condition. One dyad in which one participant identified the relationship as a friendship and the other participant identified the relationship as a stranger relationship was excluded from the analyses. Self-reported closeness scores differed significantly by relationship type, $F(2,52)=192.29, p<.001$, partial $\eta^{2}=$.88. Consistent with the manipulation, romantic relationships reported greater closeness $(M=8.83, S D=$ $0.51)$ than did friends $(M=6.57, S D=1.98), t(52)=5.64$, $p<.001$; and friends reported greater closeness than did strangers $(M=1.11, S D=0.32), t(52)=13.67, p<.001$.

To ensure consistency in water temperature across conditions, we also compared water temperature at the start of the session by touch condition and relationship condition. The average water temperature at the start of each session was $38.43{ }^{\circ} \mathrm{F}(S D=3.25)$, and did not differ by touch condition, $F(2,28)=0.52, p=.60$; relationship condition, $F(2,28)=2.12, p=.14$; or by their interaction, $F(4,28)=0.35, p=.84$.

\subsection{Hypotheses and Research Question}

The first hypothesis predicted that receiving interpersonal touch has a protective effect on pain sensitivity, and the second hypothesis predicted that the protective effect is greater for romantic relationships than for other relationships. The research question asked whether the protective effect of touch varies by the sex of the participant. 
Pain sensitivity was examined in an analysis of variance (ANOVA) with touch condition (touch, presence, absence) and relationship condition (romantic, friend, stranger) as independent factors. Participant sex was also included as an independent factor because women $(M=4.54$, $S D=1.75)$ differed significantly from men $(M=3.65$, $S D=1.30)$ in their pain sensitivity, $t(51)=-2.11, p$ $($ two-tailed $)=.04$. Multiple variables were examined for potential inclusion as covariates, including trait affection level, loneliness, opposite-sex touch avoidance, the Big-5 personality traits (extraversion, agreeableness, conscientiousness, neuroticism, and openness), body mass index, water temperature, pain threshold duration (time elapsed between start of session and indication of pain), and age. None of these variables was significantly correlated with pain sensitivity, so none was included as a covariate in the ANOVA.

The ANOVA produced significant main effects for touch condition, $F(2,35)=3.35, p=.046, \eta^{2}=.16$; and for participant sex, $F(1,35)=8.75, p=.006, \eta^{2}=.20$. Both main effects were rendered uninterpretable, however, by their inclusion on two significant disordinal interactions: a significant touch-by-relationship interaction, $F(4,35)=3.86, p=.011, \eta^{2}=.31$; and a significant touch-by-sex interaction, $F(2,35)=10.21, p<.0015$, $\eta^{2}=.37$.

Means and standard deviations for pain sensitivity by relationship condition and touch condition appear in Table 1. In response to $\mathrm{H} 1$ and $\mathrm{H} 2$, the pattern of means reveals a protective effect of touch that is different for romantic partners than for friends or strangers. Specifically, receiving touch allows romantic partners to tolerate a higher degree of pain intensity before identifying it as painful, compared to the mere presence or absence of their romantic partner. In contrast, both presence and absence allow both friends and strangers to tolerate a higher degree of pain intensity before identifying it as painful, compared to receiving touch. H2 is supported, and H1 is supported for romantic partners but not for strangers and friends.

Table 1. Means and Standard Deviations for Pain Sensitivity by Relationship Condition and Touch Condition

\begin{tabular}{cccc}
\hline Condition & Romantic & Friend & Stranger \\
\hline Touch & $4.33(1.63)$ & $3.17(0.98)$ & $3.43(1.40)$ \\
Presence & $4.00(0.89)$ & $5.50(1.26)$ & $3.83(1.33)$ \\
Absence & $3.67(1.63)$ & $4.33(1.63)$ & $4.80(2.59)$ \\
\hline
\end{tabular}

Table 2. Means and Standard Deviations for Pain Sensitivity by Touch Condition and Participant Sex

\begin{tabular}{ccc}
\hline Condition & Male & Female \\
\hline Touch & $3.38(1.06)$ & $3.70(1.64)$ \\
Presence & $4.61(1.30)$ & $4.33(1.58)$ \\
Absence & $3.00(1.05)$ & $6.00(1.29)$ \\
\hline
\end{tabular}

Means and standard deviations for pain sensitivity by touch condition and participant sex appear in Table 2. In response to RQ1, the pattern of means reveals a different pattern for women and men. Specifically, women were able to tolerate the highest degree of pain intensity before identifying it as painful when they were alone (absence condition) but were able to tolerate the lowest degree of pain intensity before identifying it as painful when they were touched. For men, presence had the most protective effect, then touch, then absence.

\section{Discussion}

Pain is a ubiquitous experience that can be ameliorated by a variety of pharmacological and behavioral therapies, as well as by interpersonal experiences of touch. Anecdotally, it is not uncommon for children or adults to prefer holding hands with a loved one while enduring a painful experience, such as receiving an injection, and some exploratory evidence indicates that young children believe such behaviors have analgesic effects [80]. It is empirically unclear, however, whether such tactile contact actually does buffer sensitivity to the painful stimulus and/or whether its buffering effects vary according to the nature of the relationship in which the touch occurs. The present experiment used an interpersonal but non-sexual form of touch-one person's hands touching another's shoulders - to determine whether receiving touch influences sensitivity to a pain induction. To rule out the possibility that a pain-buffering effect of touch is attributable only to the presence of a partner, rather than the touch itself, the touch condition was compared both to a control condition in which participants were alone and to a comparison condition in which a partner was present but not touching a participant. The study also compared romantic partners, platonic friends, and strangers to determine whether the nature of the relationship is consequential in the painameliorating effects of touch.

Consistent with predictions, touch-compared to presence and absence-was associated with a pain-buffering effect, but only for romantic partners. Specifically, participants were able to tolerate a higher intensity of pain before calling the stimulus painful when touched by a romantic partner than when their romantic partner was absent or was present without touching. The opposite was true for strangers and friends, however: Participants were able to tolerate a lower intensity of pain before calling the stimulus painful when touched by a stranger or friend than when their stranger or friend was absent or was present without touching. We also identified systematic differences related to the sex of the participant. Specifically, being alone during the pain induction was associated with the lowest sensitivity (i.e., ability to tolerate the highest pain intensity before calling it painful) for women, but the highest sensitivity for men. Conversely, women's sensitivity was highest when they were touched, suggesting that touch may be more beneficial in terms of pain management for men than for women.

Given the robust empirical literature on the benefits of touch for pain, it may be less remarkable to find an association between touch and pain sensitivity than to find that such an association varies systematically by relationship type. It is consistent with previous work, such as Coan et al.'s study, that the benefit of touch was most pronounced in romantic partnerships, yet simultaneously inconsistent with such work that touch in other relationships was not beneficial [67]. Coan et al. reported that even touch received from a stranger was superior to the absence of touch in terms of threat-related stress, and this is 
inconsistent with the present finding that touch received from strangers and friends was associated with negative outcomes (not just less-positive outcomes) for pain. (It is worth noting that the outcomes in the current study and in Coan et al.'s study were not identical, which may partially account for this discrepancy.)

That the nature of the relationship matters to the benefits of an interpersonal behavior such has touch has long been recognized in the literature on affectionate behavior, however. Affection exchange theory [73] provides that the same affectionate behavior-such as a kiss or hug-can be stress-alleviating in one relationship (such as between intimates) yet stress-inducing in another (such as between strangers), and empirical investigations have helped illuminate the conditions under which an affectionate touch or expression might be received negatively instead of positively [81,82,83]. Although the current investigation demonstrated that touch received by a friend or stranger was, in fact, detrimental to pain sensitivity, further investigation is certainly warranted before declaring touch in these relationships to be wholly non-therapeutic.

\subsection{Implications}

The current findings have implications for the management of pain in clinical practice, one being that recruiting a patient's romantic partner to administer touch during a painful medical procedure may help the patient tolerate a greater level of sensory intensity before experiencing it as painful, reducing the discomfort of the procedure. Several studies reviewed above similarly show that touch received by medical professionals can buffer pain sensitivity $[39,40]$, yet no study of which we are aware has previously demonstrated the same for touch received by romantic partners.

For painful medical procedures_-such as an injection, a bone marrow extraction, or an arterial blood gas test-a supportive touch from a romantic partner may actually be more effective than an analogous touch from a medical professional in ameliorating pain, although that comparison remains to be verified empirically. The present findings suggest, however, that receiving touch from a friend or a stranger does not confer the same pain-buffering benefits. In fact, touch from a friend or stranger may even heighten sensitivity to a painful stimulus.

\subsection{Strengths and Limitations}

The study benefited from certain methodological strengths, including a prescreening process that excluded participants who had previously been diagnosed with or treated for fibromyalgia. Because fibromyalgia is defined, in part, by heightened pain sensitivity [84], inclusion of fibromyalgia patients would not have illuminated the experience of pain for an average, non-clinical population, which was our goal. The experimental nature of the study allowed us to standardize the pain induction-cold pressor, in this case-and we would argue that our operational definition of pain sensitivity (the magnitude of pain present when the stimulus becomes painful) is more ecologically meaningful than simply the amount of elapsed time before pain is induced.
Nonetheless, the study was also limited in some important ways. For one, the inclusion and exclusion criteria guaranteed a relatively healthy sample, meaning that the findings may not generalize to those with clinical disorders. Furthermore, we included only opposite-sex encounters and exposed participants to only one type of pain stimulus. Whether touch received from a same-sex other would have the same effects, and/or whether the effects would manifest for heat, pressure, ischemic, or other forms of pain, are questions that remain to be tested. Similarly, as discussed below, we tested only one type of touch, and it is possible that a more intimate tactile behavior may produce different effects. All of these possibilities represent fertile directions for future empirical efforts.

Some may consider the self-report nature of our pain sensitivity measure to be a methodological limitation. On the contrary, the inherently subjective nature of pain dictates that self-report is the most accurate measurement strategy available. Whereas a physiological outcome, such as a change in blood pressure or cortisol level, could index the body's response to a pain induction, such outcomes would not serve as surrogates for the level of pain being experienced. Because pain is always subjective, the only accurate way to measure an individual's pain level is to ask the person how much pain he or she is experiencing, as we did.

\subsection{Conclusion}

Despite its limitations, this study clearly demonstrates that touch has an effect on pain sensitivity that is relationship-dependent. Besides determining whether this effect replicates in same-sex encounters and with other forms of pain induction, future research could benefit from examining a wider variety of touch behaviors. We chose a hands-on-shoulders gesture because it was straightforward, was relatively non-invasive, and did not have overtly sexual or romantic implications, but for the sake of external validity, other, more common forms of interpersonal touch-such as handholding, kissing, or an arm around the shoulder-should be examined for their potential effects on pain sensitivity.

\section{References}

[1] U.S. National Institutes of Health, "NIH analysis shows Americans are in pain,” Available: https://nccih.nih.gov/news/press/08112015. [Accessed Mar. 8, 2017].

[2] U.S. National Centers for Health Statistics, "Chartbook on trends in the health of Americans 2006, special feature: Pain,” Available: http://www.cdc.gov/nchs.data.hus.hus06.pdf. [Accessed Mar. 8, 2017].

[3] American Pain Foundation, "New survey reveals the devastating impact of chronic pain on the lives of patients: Patients expressed strong desire for new options to help manage their pain,” Available: http://www.prnewswire.com/news-releases/new-survey-revealsthe-devastating-impact-of-chronic-pain-on-the-lives-of-patients56134822.html. [Accessed Mar. 8, 2017].

[4] Nils, J., Girbés, E. L., Lundberg, M., Malfliet, A., and Sterling, M, "Exercise therapy for chronic musculoskeletal pain: Innovation by altering pain memories,” Manual Therapy, 20, 216-220. 2015.

[5] Cohen, S., Janicki-Deverts, D., Turner, R. B., and Doyle, W. J, "Does hugging provide stress-buffering social support? A study of susceptibility to upper respiratory infection and illness," Psychological Science, 26, 135-147. 2015. 
[6] Ditzen, B., Neumann, I. D., Bodenmann, G., von Dawans, B., Turner, R. A., Ehlert, U., and Heinrichs, M, "Effects of different kinds of couple interaction on cortisol and heart rate responses to stress in women," Psychoneuroendocrinology, 32, 565-574. 2007.

[7] Floyd, K., Pauley, P. M., and Hesse, C, "State and trait affectionate communication buffer adults' stress reactions," Communication Monographs, 77, 618-636. 2010.

[8] Floyd, K., and Riforgiate, S, "Affectionate communication received from spouses predicts stress hormone levels in healthy adults," Communication Monographs, 75, 351-368. 2008.

[9] Light, K. C., Grewen, K. M., and Amico, J. A, "More frequent partner hugs and higher oxytocin levels are linked to lower blood pressure and heart rate in premenopausal women," Biological Psychology, 69, 5-21. 2005.

[10] Cohen, S., Doyle, W. J., Skoner, D. P., Rabin, B. S., and Gwaltney, J. M, "Social ties and susceptibility to the common cold," Journal of the American Medical Association, 277, 1940-1944. 1997.

[11] International Association for the Study of Pain, IASP Taxonomy, Available: http://www.iasp-pain.org/Taxonomy [Accessed Mar. 20, 2017].

[12] Wade, J. B., Price, D. D., Hamer, R. M., Schwartz, S. M., and Hart, R. P, "An emotional component analysis of chronic pain," Pain, 40, 303-310. 1990.

[13] Wong, D. L., and Baker, C. M, "Smiling face as anchor for pain intensity scales," Pain, 89, 295-297. 2001.

[14] Price, D. D., Bush, F. M., Long, S., and Harkins, S. W, "A comparison of pain measurement characteristics of mechanical visual analogue and simple numerical rating scales,” Pain, 56, 217-226. 1994.

[15] Edens, J. L., and Gil, K. M, "Experimental induction of pain: Utility in the study of clinical pain," Behavior Therapy, 26, 197-216. 1995.

[16] Rhudy, J. L., and Meagher, M. W, "Fear and anxiety: Divergent effects on human pain thresholds," Pain, 84, 65-75. 2000.

[17] Streff, A., Kuehl, L. K., Michaux, G., and Anton, F, "Differential physiological effects during tonic painful hand immersion tests using hot and ice water," European Journal of Pain, 14, 266-272. 2010.

[18] Hanssen, M. J., Vancleef, L. M. G., Vlaeyen, J. W. S., and Peters, M. L, "More optimism less pain! The influence of generalized and pain-specific expectations on experienced cold-pressor pain," Journal of Behavioral Medicine, 37, 47-58. 2014.

[19] Fillingim, R. B., Maixner, W., Girdler, S. S., Light, K. C., Harris, M. B., Sheps, D. S., and Mason, G. A, "Ischemic but not thermal pain sensitivity varies across the menstrual cycle," Psychosomatic Medicine, 59, 512-520. 1997.

[20] Bär, K.-J., Brehm, S., Boettger, M. K., Boettger, S., Wagner, G., and Sauer, H, "Pain perception in major depression depends on pain modality," Pain, 117, 97-103. 2005.

[21] Slade, G. D., Sanders, A. E., Ohrbach, R., Fillingim, R. B. Dubner, R., Gracely R. H., Bair, E., Maixner, W., and Greenspan, J. D, "Pressure pain thresholds fluctuate with, but do not usefully predict, the clinical course of painful temporomandibular disorder," Pain, 155, 2134-2143. 2014.

[22] Leventhal, L., Brandt, M. R., Cummons, T. A., Piesla, M. J., Rogers, K. E., and Harris, H. A, "An estrogen receptor- $\beta$ agonist is active in models of inflammatory and chemical-induced pain," European Journal of Pharmacology, 553, 146-148. 2006.

[23] Hart, S., Field, T., Hernandez-Reif, M., Nearing, G., Shaw, S., Schanberg, S., and Kuhn, C, "Anorexia nervosa symptoms are reduced by massage therapy," Eating Disorders: The Journal of Treatment \& Prevention, 9, 289-299. 2001

[24] Field, T., Henteleff, T., Hernandez-Reif, M., Martinez, E. Mavunda, K., Kuhn C., and Schanberg, S, "Children with asthma have improved pulmonary functions after massage therapy," Journal of Pediatrics, 132, 854-858. 1998.

[25] Schachner, L., Field, T., Hernandez-Reif, M., Duarte, A., and Krasnegor, J, "Atopic dermatitis symptoms decrease in children following massage therapy,” Pediatric Dermatology, 15, 390-395. 1998.

[26] Field, T., Hernandez-Reif, M., LaGreca A., Shaw, K., Schanberg, S., and Kuhn, C, "Massage therapy lowers blood glucose levels in children with diabetes mellitus,” Diabetes Spectrum, 10, 237-239. 1997.

[27] Diego, M. A., Field, T., Hernandez-Reif, M., Shaw, K., Friedman, L., and Ironson, G, "HIV adolescents show improved immune function following massage therapy," International Journal of Neuroscience, 106, 35-45. 2001.

[28] Hernandez-Reif, M., Field, T., Krasnegor, J., Theakston, H., Hossain, Z., and Burman, I, "High blood pressure and associated symptoms were reduced by massage therapy," Journal of Bodywork and Movement Therapies, 4, 31-38. 2000.

[29] Field, T., Cullen, C., Diego, M., Hernandez-Reif, M., Sprinz, P., Beebe, K., Kissell, B., and Bango-Sanchez, V, "Leukemia immune changes following massage therapy," Journal of Bodywork and Movement Therapies, 3, 1-5. 2001.

[30] Hernandez-Reif, M., Field, T., and Hart, S, "Smoking cravings are reduced by self-massage,” Preventive Medicine, 28, 28-32. 1999.

[31] Hernandez-Reif, M., Field, T., Largie, S., Cullen, C., Beutler, J., Sanders, C., Weiner, W., Rodriguez-Bateman, D., Zelaya, L., Schanber, S., and Kuhn, C, "Parkinson's disease symptoms are differentially affected by massage therapy versus progressive muscle relaxation: A pilot study," Journal of Bodywork and Movement Therapies, 6, 177-182. 2002.

[32] Field, T., and Hernandez-Reif, M, "Sleep problems in infants decrease following massage therapy," Early Child Development and Care, 168, 95-104. 2001.

[33] Lindgren, L., Rundgren, S., Winsö, O., Lehtipalo, S., Wiklund, U., Karlsson, M., Stenlund, H., Jacobsson, C., and Brulin, C, "Physiological responses to touch massage in healthy volunteers," Autonomic Neuroscience, 158, 105-110. 2010.

[34] Frey Law, L. A., Evens, S., Knudtson, J., Nus, S., Scholl, K., and Sluka, K. A, "Massage reduces pain perception and hyperalgesia in experimental muscle pain: A randomized, controlled trial," The Journal of Pain, 9, 714-721. 2008.

[35] Mitchinson, A. R., Kim, H. M., Rosenberg, J. M., Geisser, M., Kirsch, M., Cikrit, D., and Hinshaw, D. B, "Acute postoperative pain management using massage as an adjuvant therapy,” Archives of Surgery, 142, 1158-1167. 2007.

[36] Kutner, J. S., Smith, M. C., Corbin, L., Hemphill, L., Benton, K., Mellis, B. K., Beaty, B., Felton, S., Yamashita, T. E., Bryant, L. L., and Fairclough, D. L, "Massage therapy versus simple touch to improve pain and mood in patients with advanced cancer: A randomized trial," Annals of Internal Medicine, 149, 369-379. 2008.

[37] Kshettry, V. R., Carole, L. F., Henly, S. J., Sendelbach, S., and Kummer, B, "Complementary alternative medical therapies for heart surgery patients: Feasibility, safety, and impact," The Annals of Thoracic Surgery, 81, 201-205. 2006.

[38] Suresh, S., Wang, S., Porfyris, S., Kamasinski-Sol, R., and Steninhorn, D. M, "Massage therapy in outpatient pediatric chronic pain patients: Do they facilitate significant reductions in levels of distress, pain, tension, discomfort, and mood alterations?” Pediatric Anesthesia, 18, 884-887. 2008.

[39] Peterson, A. M., Cline, R. J. W., Foster, T. S., Penner, L. A., Parrott, R. L., Keller, C. M., Naughton, M. C., Taub, J. W., Ruckdeschel, J. C., and Albrecht, T. L, 'Parents' interpersonal distress and touch behavior and child pain and distress during painful pediatric oncology procedures," Journal of Nonverbal Behavior, 31, 79-97. 2007.

[40] Weekes, D. P., Kagan, S. H., James, K., and Seboni, N, "The phenomenon of hand holding as a coping strategy in adolescents experiencing treatment-related pain,” Journal of Pediatric Oncology Nursing, 10, 19-25. 1993.

[41] Mok, E., and Woo, C. P, "The effects of slow-stroke back massage on anxiety and shoulder pain in elderly stroke patients," Complementary Therapies in Nursing and Midwifery, 10, 209-216. 2004.

[42] Cong, X., Cusson, R. M., Walsh, S., Hussain, N., Ludington-Hoe, S. M., and Zhang, D, "Effects of skin-to-skin contact on autonomic pain responses in preterm infants," The Journal of Pain, 13, 636-645. 2012.

[43] Cong, X., Cusson, R. M., Hussain, N., Zhang, D., and Kelly, S. P, "Kangaroo care and behavioral and physiological pain responses in very-low-birth-weight twins: A case study,” Pain Management Nursing, 13, 127-138. 2012.

[44] Herrington, C. J., and Chiodo, L. M, "Human touch effectively and safely reduces pain in the newborn intensive care unit," Pain Management Nursing, 15, 107-115. 2014.

[45] Hogendoorn, H., Kammers, M., Haggard, P., \& Verstraten, F, "Self-touch modulates the somatosensory evoked P100," Experimental Brain Research, 233, 2845-2858. 2015. 
[46] Floyd, K, “Affection deprivation is associated with physical pain and poor sleep quality,” Communication Studies, 67, 379-398. 2016.

[47] Melzack, R., and Wall, P. D, "Pain mechanisms: A new theory,” Science, 150, 971-979. 1967.

[48] Le Bars, D, "The whole body receptive field of dorsal horn multireceptive neurons,” Brain Research Reviews, 40, 29-44. 2002.

[49] Mancini, F., Nash, T., Iannetti, G. D., and Haggard, P, "Pain relief by touch: A quantitative approach,” Pain, 155, 635-642. 2014.

[50] Dunbar, R. I. M, “The social role of touch in humans and primates: Behavioural function and neurobiological mechanisms," Neuroscience \& Biobehavioral Reviews, 34, 260-268. 2010.

[51] Uvnäs-Moberg, K., Arn, I., and Magnusson, D, "The psychobiology of emotion: The role of the oxytocinergic system," International Journal of Behavioral Medicine, 12, 59-65. 2005.

[52] Miranda-Cardenas, Y., Rojas-Piloni, G., Martínez-Lorenzana, G., Rodríguez-Jiménez, J., López-Hidalgo, M., Freund-Mercier, M. J., and Condés-Lara, M, "Oxytocin and electrical stimulation of the paraventricular hypothalamic nucleus produce antinociceptive effects that are reversed by an oxytocin antagonist," Pain, 122, 182-189. 2006

[53] Uvnäs-Moberg, K, "Neuroendocrinology of the mother-child interaction,” Trends in Endocrinology and Metabolism, 7, 126-131. 1996.

[54] Husslein, P., Fuchs, A.-R., and Fuchs, F, "Oxytocin and the initiation of human parturition: I. Prostaglandin release during induction of labor by oxytocin," American Journal of Obstetrics and Gynecology, 141, 688-693. 1981.

[55] Murphy, M. R., Seckl, J. R., Burton, S., Checkley, S. A., and Lightman, S. L, "Changes in oxytocin and vasopressin secretion during sexual activity in men,” Journal of Clinical Endocrinology and Metabolism, 65, 738-741. 1990.

[56] Richard, P., Moos, F., and Freund-Mercier, M. J, “Central effects of oxytocin,” Physiological Review, 71 (2), 331-370. 1991.

[57] Heinrichs, M., Baumgartner, T., Kirschbaum, C., and Ehlert, U, "Social support and oxytocin interact to suppress cortisol and subjective responses to psychosocial stress," Biological Psychiatry, 54, 1389-1398. 2003.

[58] Holt-Lunstad, J., Birmingham, W., A., and Light, K. C, "Influence of a "warm touch" support enhancement intervention among married couples on ambulatory blood pressure, oxytocin, alpha amylase, and cortisol,” Psychosomatic Medicine, 70, 976-985. 2008.

[59] Turner, R. A., Altemus, M., Enos, T., Cooper, B., and McGuinness, T, "Preliminary research on plasma oxytocin in normal cycling women: Investigating emotion and interpersonal distress,” Psychiatry, 62, 97-113. 1999.

[60] Henricson, M., Berglund, A. L., Määttä, S., Ekman, R., and Segesten, K, "The outcome of tactile touch on oxytocin in intensive care patients: A randomised controlled trial," Journal of Clinical Nursing, 17, 2624-2633. 2008.

[61] Ågren, G., Lundeberg, T., Uvnäs-Moberg, K., and Sato, A, “The oxytocin antagonist 1-deamino-2-- -Tyr-(Oet)-4-Thr-8-Orn-oxytocin reverses the increase in the withdrawal response latency to thermal, but not mechanical nociceptive stimuli following oxytocin administration or massage-like stroking in rats," Neuroscience Letters, 187, 49-52. 1995.

[62] Stefano, G. B., Goumon, Y., Casares, F., Cadet, P., Fricchione, G. L., Rialas, C., Peter, D., Sonetti, D., Guarna, M., Welters, I. D. and Bianchi, E, "Endogenous morphine,” Trends in Neurosciences, 23, 436-442. 2000.

[63] Nelson, E. E., and Panksepp, J, "Brain substrates of infant-mother attachment: Contributions of opioids, oxytocin, and norepinephrine,” Neuroscience \& Biobehavioral Reviews, 22, 437-452. 1998.

[64] Howlett, T. A., Tomlin, S., Ngahfoong, L., Rees, L. H., Bullen, B. A., Skrinar, G. S., and McArthur, J. W, "Release of $\beta$-endorphin and met-enkephalin during exercise in normal women: Response to training,” British Medical Journal, 288, 1950-1952. 1984.
[65] Zacur, H. A., Chapanis, N. P., Lake, C. R., Ziegler, M., and Tyson, J. E, "Galactorrhea-amenorrhea: Psychological interaction with neuroendocrine function," American Journal of Obstetrics and Gynecology, 125, 859-862. 1976.

[66] Weinstein, D., Launay, J., Pearce, E., Dunbar, R. I. M., and Stewart, L, "Singing and social bonding: Changes in connectivity and pain threshold as a function of group size," Evolution \& Human Behavior, 37, 152-158. 2016.

[67] Coan, J. A., Schaefer, H. S., and Davidson, R. J, "Lending a hand: Social regulation of the neural response to threat," Psychological Science, 17, 1032-1039. 2006.

[68] Fillingim, R. B, "Sex, gender, and pain: Women and men really are different," Current Review of Pain, 4, 24-30. 2000.

[69] Kim, H., Neubert, J. K., Mighel, A. S., Xu, K., Krishnaraju, R. K., Iadarola, M. J., Goldman, D., and Dionne, R. A, "Genetic influence on variability in human acute experimental pain sensitivity associated with gender, ethnicity and psychological temperament,” Pain, 109, 488-496. 2004.

[70] DeWall, C. N., and Baumeister, R. F, “Alone but feeling no pain: Effects of social exclusion on physical pain tolerance and pain threshold, affective forecasting, and interpersonal empathy," Journal of Personality and Social Psychology, 91, 1-15. 2006.

[71] Vaegter, H. B., Handberg, G., and Graven-Nielsen, T, "Hypoalgesia after exercise and the cold pressor test is reduced in chronic musculoskeletal pain patients with high pain sensitivity," The Clinical Journal of Pain, 32, 58-69. 2016.

[72] Floyd, K, "Human affection exchange: V. Attributes of the highly affectionate,” Communication Quarterly, 50, 135-152. 2002.

[73] Floyd, K, Communicating affection: Interpersonal behavior and social context, Cambridge University Press, Cambridge, 2006.

[74] Russell, D. W, "UCLA Loneliness Scale (version 3): Reliability, validity, and factor structure,” Journal of Personality Assessment, 66, 20-40. 1996.

[75] Russell, D., Peplau, L. A., and Ferguson, M. L, "Developing a measure of loneliness," Journal of Personality Assessment, 42, 290-294. 1978.

[76] John, O. P., Naumann, L. P., and Soto, C. J, "Paradigm shift to the integrative Big-Five trait taxonomy: History, measurement, and conceptual issues,” in O. P. John, R. W. Robins, and L. A. Pervin (Eds.), Handbook of personality: Theory and research, Guilford Press, New York, 2008, 114-158.

[77] John, O. P., and Srivastava, S. "The Big-Five trait taxonomy: History, measurement, and theoretical perspectives,” in L. A. Pervin and O. P. John (Eds.), Handbook of personality: Theory and research, Guilford Press, New York, 1999, 102-138.

[78] Andersen, P. A., and Leibowitz, K, "The development and nature of the construct of touch avoidance," Journal of Nonverbal Behavior, 3, 89-106. 1978.

[79] Andersen, P. A, “The touch avoidance measure," in V. L. Manusov (Ed.), The sourcebook of nonverbal measures: Going beyond words, Sage, Thousand Oaks, 2005, 57-65.

[80] Franck, L. S., Sheikh, A., and Oulton, K, "What helps when it hurts: Children's views on pain relief,” Child: Care, Health and Development, 34, 430-438. 2008.

[81] Erbert, L. A., and Floyd, K, “Affectionate expressions as facethreatening acts: Receiver assessments," Communication Studies, 55, 230-246. 2004.

[82] Floyd, K., and Burgoon, J. K, "Reacting to nonverbal expressions of liking: A test of interaction adaptation theory," Communication Monographs, 66, 219-239. 1999.

[83] Floyd, K., and Voloudakis, M, "Affectionate behavior in adult platonic friendships: Interpreting and evaluating expectancy violations,” Human Communication Research, 25, 341-369. 1999.

[84] Petzke, F., Clauw, D. J., Ambrose, K., Khine, A., and Gracely, R. $\mathrm{H}$, "Increased pain sensitivity in fibromyalgia: Effects of stimulus type and mode of presentation,” Pain, 105, 403-413. 2003. 